\title{
IMAGE FUSION ANALYSIS FOR HYPERSPECTRAL DATA
}

\author{
Y.Sai Praveen ${ }^{1}$, A.Kiranmai2, Iyyanki. V. Murali Krishna ${ }^{3}$, K.Nikitha ${ }^{4}$ \\ ${ }^{1}$ Dept of CSE, GITAM University, Hyderabad \\ ${ }^{2}$ Dept of CSE, GITAM University, Hyderabad \\ ${ }^{3}$ Dr. Raja Ramanna Distinguished fellow, Research center Imarat, DRDO \\ ${ }^{4}$ Assistant Professor, Dept of CSE, GITAM University, Hyderabad
}

\begin{abstract}
The hyperspectral sensors provide the images with hundreds of narrow contiguous spectral channels which provide plenty of spectral information and with this information; we can achieve high accuracy in classification problems and detection or identification of targets. However, the hyperspectral images are high in spectral resolution but low in spatial resolution. So in order to enhance the image with both spectral and spatial resolution, we support image fusion. In this paper, we have done image fusion along with preprocessing techniques such as radiometric and atmospheric corrections. The high dimensionality of the hyperspectral images may increase the computational complexity. We applied a dimensionality reduction technique that is Principal component analysis to reduce the number of bands that we consider for processing, and then performed the Image sharpening to enhance the spatial resolution. So the resulting will be high in both spectral and spatial resolution, and analysis of these image fusion techniques is also studied.
\end{abstract}

Keywords: Dimensionality Reduction, Hyperspectral, Principal Component Analysis,, Minimum Noise Fraction, Image Fusion, Gram Schmidt Pan sharpening.

\section{INTRODUCTION}

In remote sensing, the Panchromatic sensors provide high spatial resolution, and multispectral and hyperspectral images provide spectral resolution. Compared to multispectral images hyperspectral images provide more spectral information. Multispectral images contain around 46 bands visible and infrared regions of the electromagnetic spectrum. Each band contains different information when compared to the other bands. The multispectral images provide more information for applications like classification and target detection than single band images. Multispectral images are better than single grey scale image. We can obtain RGB composite of the image by using the channels which are captured in Red, Green, Blue regions of the electromagnetic spectrum. But in some applications, it is observed that the spectral information provides by multispectral images may not be sufficient for differentiating the materials which are having the somewhat similar spectral signature. In those cases, the multispectral images cannot discriminate between the two different materials. So by using Hyperspectral images we can overcome this problem [3], [5].

Hyperspectral sensor Images contains hundreds of narrow spectral channels or bands taken along the electromagnetic spectrum covering visible, near-infrared and short-wave infrared regions. With this hyperspectral images provide more spectral information compared to multispectral images which is very much useful in many remote sensing applications. With this, we can get more accuracy in applications like classification and target detection. In this paper, we have used Hyperion data which is a hyperspectral sensor which contains 242 spectral bands ranging from $355.59-2577.08 \mathrm{~nm}$. In hyperspectral images only some specific number of bands useful because of the small signal to noise ratio and most of the bands in these images contain redundant and some band may get affected by the atmospheric effects. So we need to consider only a set of bands for processing in order to reduce the computational cost. Even though the hyperspectral image provides very richer spectral information but these images arelack in spatial resolution. So with the pan sharpening technique, we can obtain the image with both spectral and spatial resolution. This is also called as image fusion. In this, we considered a panchromatic image which is single band image which is high in spatial resolution and we try to fuse this PAN image with the corresponding Hyperspectral image. Sothe resulting image contains both spectral and spatial resolution. In this paper we have studied some of these techniques of image fusion[1], [2], [3].

\section{LITERATURE}

Pohl and Genderen Van, 1998 proposed that image fusion is a method to combine the different source imagery using the image sharpening techniques. According to Pohl and Genderen High-Resolution and Hyperspectral Data Fusion for Classification Van, 1998, the main objectives of image fusion are to improve the spectral and spatial quality of the images, and also improve atmospheric, radiometric and geometric corrections which enhance certain features that are not visible in the images, now replace the processed data, correct the data sets for the improving classification, detection of target. 
Fusion methods can be divided into two categories: first method simultaneously takes into account all bands in the fusion process e.g. Hue- Saturation-Value transformation, Principle-Component transformation, Gram-Schmidt transformation technique; the second category deal separately with the spatial information and each spectral band e.g. Brovey transformation, High-Pass-Filter transformation technique.

The main objective of the study was the investigation of the spectral properties and the statistical analysis of the fused images with the Hyperion data. The results of the fusion show that the GST [4] and PCT [4] have almost similar in image quality the statistics as compared to draw a conclusion that which is the best fusion technique for the desired hyperspectral data. The high-frequency band spectrum values are compared with the low-frequency band spectrum to improve the accuracy. Different statistical parameters like the standard deviation, mean, median, and mode, maximum, minimum values of the Hyperion data and the two fused images (GST \& PCT) were compared for the analysis.

\section{STUDY AREA AND DATASETS}

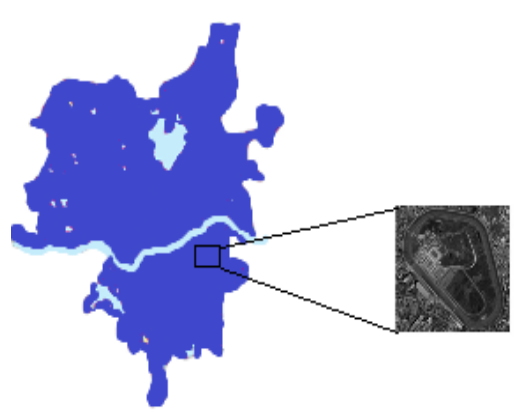

Fig. 1 Study Area

Study area is part of Hyderabad city .It falls in the longitude and latitude of $17.3700^{\circ} \mathrm{N}$ and $78.4800^{\circ} \mathrm{E}$. The input datasets are EO-1 Hyperion which is a hyperspectral image and orbview-3 which is a panchromatic image. TheHyperion image is having 30-meter spatial resolution and having 242 bands. The orview-3 image is having 1-meter spatial resolution contains only one band which is a grey scale image. The dataset is atmospherically and radiometrically corrected. The hyperspectral data is geometrically corrected such that both the data sets have same geometrical projections. Co-registration has applied to the datasets.

\section{PREPROCESSING OF DATA}

The images collected hyperspectral sensor cannot be applied remote sensing applications directly. The hyperspectral data that we get can get affected by the atmospheric affects such as water vapor, haze etc. which reduce the spectral reflectance that is received by the sensor. They must be preprocessed in order to convert the radiance that we get from the data to reflectance and to reduce the noise, we use the data for further processing. The raw data that we get from sensor might not be geometrically correct. With respect to hyperspectral data, some of the bands may contain only noise. So we need to remove those bands form processing. So preprocessing of the hyperspectral images is necessary in order to remove these undesirable effects which may reduce the accuracy of the results[5].

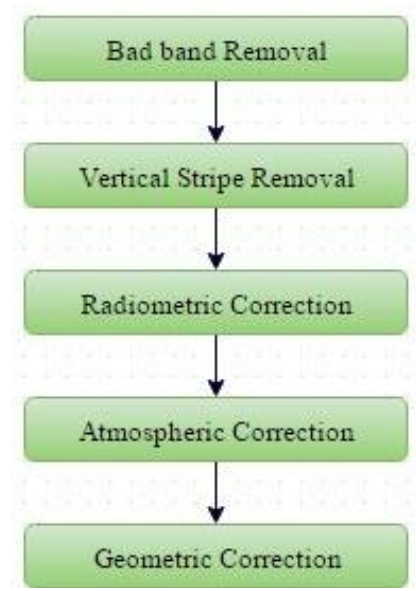

Fig. 2 Pre-processing of Hyperspectral images

The hyperspectral data collected by the sensor contains some zero and noisy bands which do not contain any valid information and sometimes may decrease the accuracy of the feature extraction and classification results. By identifying and removing this bad bands from the data to be processed will reduce the dimensionality of the data.

Some bands in the hyperspectral images contain a vertical stripe which is known as "striping" effect. These are black lines in the images which do not contain any useful information. They are the result of the erroneous functioning of the detector in the sensor. As a pre-processing step, we should check each band for striping effect and they should be minimized. It can be done by weighted averaging technique. In this, we take the average of neighbor pixel DN values and we replace this value with affected column pixel values.

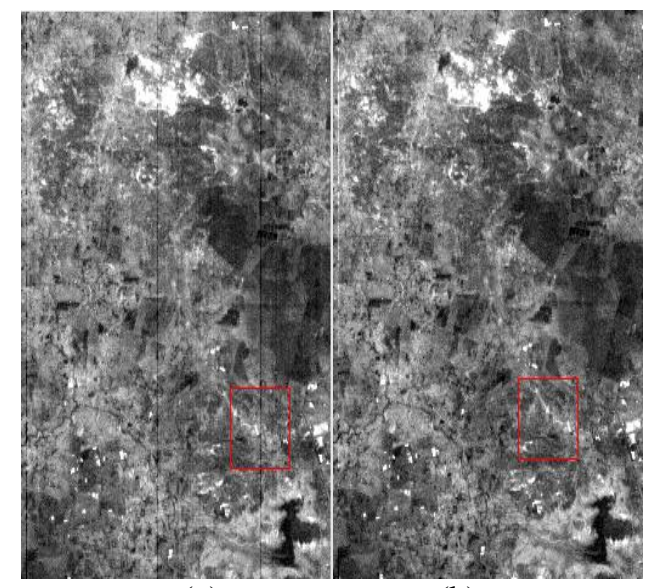

(a)

(b)

Fig. 3: (a) Before vertical Stripe Correction (b) After Vertical stripe correction

Radiometric correction is applied to the remotely sensed data to improve the fidelity of the brightness value. The brightness value may be attenuated or reduced because of 
some errors or some atmospheric effects. These effects may reduce the interpretation and analyzing capability. In order recover from these radiometric distortions we have to apply radiometric correction to the data.

Before the reflectance or the signal from the earth surface reached by the sensor this signal get affected by the various atmospheric effects such as water vapor absorption, aerosols which attenuate the signal that is received by the sensor. Correction of these effects is required in order to get surface reflectance spectra from imaging radiance data. Approximately the region between 0.4 and $2.5 \mu \mathrm{m}$ is affected by atmospheric water vapor absorption Because of this Atmospheric correction is a necessary pre-processing step for the most Hyperspectral Imagery (HSI) data analysis approaches.

Geometric corrections must be done for the both data sets such that they have same geometric projections. The data can be corrected geometrically by having sensor altitude and position.

\section{DIMENSIONALITY REDUCTION}

Hyperspectral images provide significant amount of spectral information which is enough for discriminating the two different materials. But these hyperspectral data contains more redundant information with respect to other bands, which increases the computational complexity in terms of Time and Size. For this reason, it is necessary to reduce the data dimensionality. Various techniques have been developed for reducing this dimensionality problem. The technique that we considered here is Principal Component Analysis.

\subsection{Principal Component Analysis (PCA)}

PCA is a dimensionality reduction technique. It is based on an orthogonal transformation. In this the components produced by Principal Component Analysis are having highest variance. It removes the correlation among the bands. The PCA technique reduces the dimensionality by producing the components with high variation and less correlation. The noise bands are segregated they are having very less Eigen value[5].

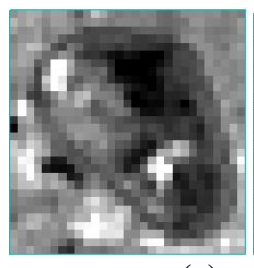

(a)

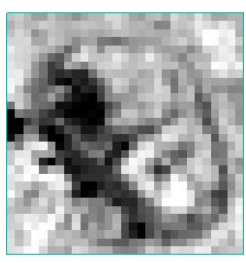

(b)

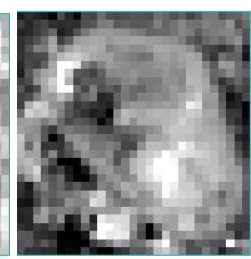

(c)

\section{Fig. 4 PCA bands (a) PCA 1 (b) PCA 2 (c) PCA 3}

The first PC band contains the largest percentage of data variance and the second $\mathrm{PC}$ band contains the second largest data variance, and so on. Table 1 show that the first band contains the highest Eigen value and the second band contains the second highest Eigen value. The last PC bands are contains more noise and very little variance compared to other bands. By comparing the Eigen values of the PCA bands we select only those bands which are having highest Eigen values compared to the all others. By increasing the number of bands we select for processing we can increase the accuracy of the results. Figure 5 represents first three bands of PCA. From the table 1 we can observe that the Eigen value of PCA 1 is having highest value.

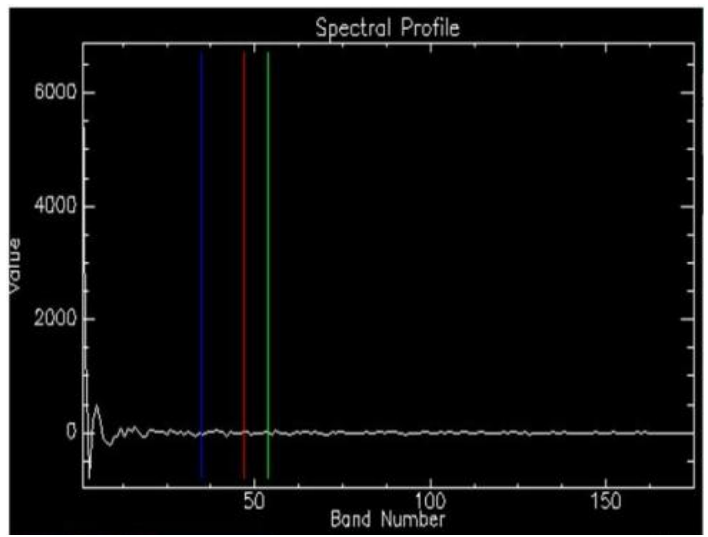

Fig. 5 Spectral Profile of PCA

\section{IMAGE FUSION}

Image fusion is a technique used to increase the spatial resolution of a hyperspectral image with high spectral resolution but with low spatial resolution. In the first step, the low-resolution data is rescaled to match the high spatial resolution and finally, fusion is achieved by adding the highfrequency content to the low spatial resolution image. The techniques that we have considered here are Gram-Schmidt [8],[9],[11], Brovey [4],[5] , PC sharpening and HSV. For selecting the good fusion technique we have to consider some accuracy measures such as SNR, RMSE error. In order to maximize the signal to noise ratio, we can apply conventional or modern dimensionality reduction technique like PCA, ICA and MNF which reduces the noise and increases the signal [3],[5],[6].

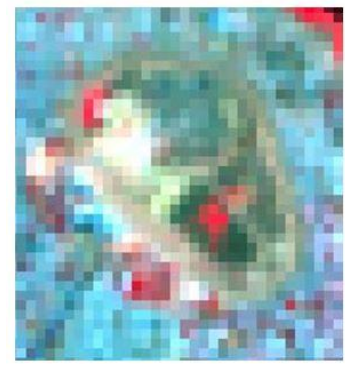

(a)

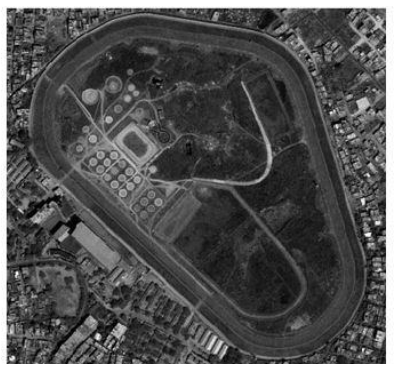

(b)
Fig. 6 Fusion Data (a) Hyperion (30 meters) (b) Orbview$3(1$ meter $)$

The methodology that we have selected for fusion of hyperspectral and panchromatic is simple and robust, where we have fused Hyperion image of $30 \mathrm{mts}$ spatial resolution with the orbview-3 image of $1 \mathrm{mts}$ spatial resolution here we can apply dimensionality reduction techniques if the data 
bands are more. Here some of the fusions that we have performed [8],[11],[13],[14].

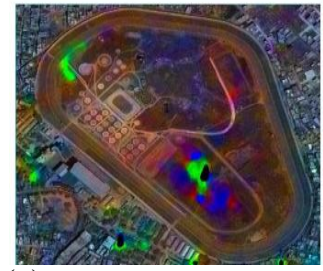

(a)

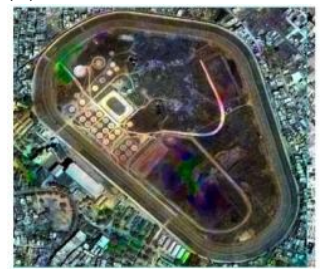

(c)

Fig. 7 Fusions (a) Brovey Fusion (b) PC Spectral Sharpening Fusion (c)HSV Fusion (d) Gram Schmidt Fusion

Table 1: SNR

\begin{tabular}{|c|c|c|c|c|}
\hline Bands & Gram & Brovey & PC sharpening & HSV \\
\hline Band 1 & 21.04010929 & 3.292651 & 7.000559469 & 1.633029154 \\
\hline Band 2 & 20.34726739 & 3.134919924 & 7.021209421 & 1.614669173 \\
\hline Band 3 & 18.5434778 & 3.145894128 & 7.015892257 & 1.569591624 \\
\hline Average & 19.97695149 & 3.191155017 & 7.012553716 & 1.605763317 \\
\hline
\end{tabular}

\section{CONCLUSION}

In this paper we have given a brief outlook of hyperspectral and panchromatic data, image fusion techniques .data preprocessing is also done up where our data required which will help to enhance the working performance of the fusion techniques .Here are some evaluation metrics are considered to select the best fusion technique in future we can increase the assessment measures for improving Image fusion techniques.

\section{REFERENCES}

[1] Y. Yang, C. Han, X. Kang and D. Han, "An Overview on Pixel-Level Image Fusion in Remote Sensing," Automation and Logistics, 2007 IEEE International Conference on, Jinan, 2007, pp. 23392344.

[2] Choi, Myungjin. "A new intensity-hue-saturation fusion approach to image fusion with a tradeoff parameter." IEEE Transactions on Geoscience and Remote sensing 44.6 (2006): 1672-1682.

[3] Li, Cunjun, et al. "Comparison of two methods of the fusion of remote sensing images with fidelity of spectral information." Geoscience and Remote Sensing Symposium, 2004. IGARSS'04. Proceedings. 2004 IEEE International. Vol. 4. IEEE, 2004.

[4] Ranchin, Thierry, and Lucien Wald. "Fusion of high spatial and spectral resolution images: the ARSIS concept and its implementation." Photogrammetric Engineering and Remote Sensing 66.1 (2000): 49-61.
[5] Wang, Zhijun, et al. "A comparative analysis of image fusion methods." Geoscience and Remote Sensing, IEEE Transactions on 43.6 (2005): 13911402.

[6] Karathanassi, V., P. Kolokousis, and S. Ioannidou. "A comparison study on fusion methods using evaluation indicators." International Journal of Remote Sensing 28.10 (2007): 2309-2341.

[7] Klonus, Sascha, and Manfred Ehlers. "Image fusion using the Ehlers spectral characteristics preservation algorithm." GIScience\& Remote Sensing 44.2 (2007): 93-116.

[8] Aiazzi, B., et al. "MS p Pan image fusion by an enhanced Gram-Schmidt spectral sharpening." (2007).

[9] Ehlers, Manfred. "Multi-image fusion in remote sensing: spatial enhancement vs. spectral characteristics preservation." Advances in Visual Computing. Springer Berlin Heidelberg, 2008. 75-84.

[10] Alimuddin, Ilham, JosaphatTetuko Sri Sumantyo, and Hiroaki Kuze. "Assessment of pan-sharpening methods applied to image fusion of remotely sensed multi-band data." International Journal of Applied Earth Observation and Geoinformation 18 (2012): 165-175.

[11] Cetin, Mufit, and NebiyeMusaoglu. "Merging hyperspectral and panchromatic image data: qualitative and quantitative analysis." International Journal of Remote Sensing 30.7 (2009): 1779-1804.

[12] Bruzzone, L., et al. "Can multiresolution fusion techniques improve classification accuracy?." Remote Sensing. International Society for Optics and Photonics, 2006.

[13] Chisense, Chembe, et al. "Pansharpening of hyperspectral images in urban areas." Proc. XXII Congr. of the Int. Society for Photogrammetry, Remote Sens 39 (2012): 8.

[14] Chen, Zhao, et al. "Fusion of hyperspectral and multispectral images: A novel framework based on generalization of pan-sharpening methods." Geoscience and Remote Sensing Letters, IEEE 11.8 (2014): 1418-1422. 